\title{
Sensitivity analysis enables comparison of how realistic morphology and other intrinsic properties influence neuronal firing Christina M Weaver*1,2,3, Georgi Gamkrelidze ${ }^{2,3,4}$, Robert Baker ${ }^{4}$ and Susan L Wearne $e^{1,2,3}$
}

\author{
Address: ${ }^{1}$ Laboratory of Biomathematics, Mount Sinai School of Medicine, New York, NY 10029, USA, ${ }^{2}$ Computational Neurobiology and \\ Imaging Center, Mount Sinai School of Medicine, New York, NY 10029, USA, ${ }^{3}$ Department of Neuroscience, Mount Sinai School of Medicine, \\ New York, NY 10029, USA and 4Department of Physiology and Neuroscience, New York University Medical School, New York, NY 10016, USA \\ Email: Christina M Weaver* - christina.weaver@mssm.edu \\ * Corresponding author
}

from Sixteenth Annual Computational Neuroscience Meeting: CNS*2007

Toronto, Canada. 7-12 July 2007

Published: 6 July 2007

BMC Neuroscience 2007, 8(Suppl 2):S20 doi:10.1 186/I47I-2202-8-S2-S20

(C) 2007 Weaver et al; licensee BioMed Central Ltd.

Several studies have shown that dendritic morphology and spatial distributions of active ion channels contribute significantly to neuronal firing dynamics and signal processing, however the relative importance and interactions between these mechanisms are not well understood. Within computational models these intrinsic properties are represented by parameters with different units and magnitudes that interact nonlinearly to simulate experimentally observed behaviors. Mathematical sensitivity analysis provides a tool to assess how strongly these parameters influence model output. For a given model neuron, the normalized sensitivity coefficient ('sensitivity') of its output to a particular parameter describes the percentage change in its output for a one percent increase in that parameter. The sensitivity magnitude indicates how much the measured output changes under the perturbation, while its sign indicates whether the output increases or decreases. One such sensitivity can be computed to each parameter; its sign and magnitude may vary for models represented by different points in a space defined by morphologic, active membrane, and passive cable parameters.

We perform this sensitivity analysis on a compartmental model comprising a soma and cylindrical active dendrite, with output measured by firing rates under current injections and by firing rate gain. Across the parameter space, sensitivity of these output variables to perturbations of dendritic length, diameter and surface area was compared with sensitivity to active and passive conductance parameters. For spontaneous firing rate, sensitivity increased most with increasing levels of persistent sodium and Atype potassium conductance, whereas for gain, sensitivity increased most as high-threshold calcium conductance decreased. Particularly in regions of space with slow calcium removal from the cytoplasm in the model cells, sensitivities of firing rates and gain to dendritic diameter and surface area were greater than to almost all active parameters.

The sensitivity analysis was extended to a model neuron from the precerebellar nucleus Area II of goldfish. Area II neurons are necessary for eye velocity storage, a mechanism that displays persistent activity after extinguishing visual or vestibular stimuli. Parameter optimization identified sets of active and passive model parameters consistent with Area II electrophysiology for a morphology obtained in vivo and traced in 3D. Sensitivities to perturbations of dendritic length, diameter and surface area were compared with those of active and passive parameters. These data indicate that, as in the simple model, there are regions of parameter space where dendritic morphology influences firing rate and gain more strongly than active conductances or passive cable parameters. 


\section{Acknowledgements}

Supported by NIH grants DC05669, NSI3742 and MH07I8I8.

Publish with Bio Med Central and every scientist can read your work free of charge

"BioMed Central will be the most significant development for disseminating the results of biomedical research in our lifetime. " Sir Paul Nurse, Cancer Research UK

Your research papers will be:

- available free of charge to the entire biomedical community

- peer reviewed and published immediately upon acceptance

- cited in PubMed and archived on PubMed Central

- yours - you keep the copyright 